\title{
GEOMETRY EFFECTS ON THE NOISE REDUCTION OF HELMHOLTZ RESONATORS
}

\author{
S.Mekid*1, M.Farooqui ${ }^{1}$, and Z.Liu $^{2}$ \\ ${ }^{1}$ King Fahd University of Petroleum and Minerals, \\ Department of Mechanical Engineering, Dhahran, 31261, Saudi Arabia \\ ${ }^{2}$ Dresser-Rand Company, Olean, NY, USA 1K3
}

\begin{abstract}
The effect of geometry shape of the Helmholtz resonator on its resonant frequency and noise attenuation capability is discussed in this paper. The theory of resonant frequency depending on the shape of the vessel of the resonator is verified analytical and numerically using COMSOL for one degree of freedom resonators. The simulation was validated experimentally and has shown very good agreements. Various shapes of the resonators were compared in arrays. A better understanding of the shape effect is shown through simulations.
\end{abstract}

Keywords: Helmholtz resonators, noise reduction, conical resonators, spherical resonators

\section{Introduction}

Excessive noise generated by compressors and other turbomachineries is a real concern in industries and refineries. The significant impact of this noise is the discomfort of the personnel working at the facility. In a couple of petroleum plants the authors have visited, the primary concern is that the noise of the compressors drowns the noise of the emergency alarms of the facility which sometime poses a serious safety issue. The next concern is the usual noise safety limitation for people working in the plant. The noise levels in compressors vary over a wide range from $70-120$ $\mathrm{dB}[1,2,3]$. As the compressor operates over its lifetime, the noise and vibration levels may expectedly increase, since centrifugal compressors are continuous flow machines and are extensively used in Saudi Arabia at crude oil processing facilities, maintenance is periodic and stopping the operation every time noise levels exceed the desired threshold can be very expensive. Currently Dresser-Rand compressors use customized Duct Resonator arrays (DR arrays) [1, 2].This solution was applied successfully to a 2528 PSIG (172 BARG) multistage centrifugal compressor on a platform in the North Sea and was shown to successfully give a reduction of up to $12 \mathrm{~dB}$. Over the last few years, Dresser Rand has revamped more than 250 centrifugal compressors, both single stage and multistage $[1,2]$, with the DR arrays. It appeared that reducing manufacturing cost can further increase the healthy margin of this product.

\section{Sources of noise in centrifugal compressors}

Noise originates from various sources within compressors. The most critical source of noise in centrifugal compressors is considered to be the blade passing frequency noise. This noise arises from the interaction between the impeller blade and the stationary diffuser vanes $[1,2,3]$. It is widely known that Blade Passing Frequency (BPF) noise components originate from the circumferential flow distortions upstream and downstream of the impeller [6]. The interaction between the impeller blades as it passes by the stationary diffuser vane causes a pressure pulsation which leads to the development of positive and negative vortices.

The interaction of these vortices as they move along the flow path creates the discrete frequency noises of the blade passing frequency. Conventionally the BPF falls between $1000 \mathrm{~Hz}$ to $4500 \mathrm{~Hz}$, usually depending on the speed of the compressor and the number of impeller blades [1].

This range falls within human hearing sensitivity which adds to the irritating nature of this noise. Although the BPF may be considered to be the most annoying aspect of compressor noise, at supersonic flow conditions another source of noise arises in the form of buzz saw noise. The BPF noise and the buzz saw noise coupled together can lead to structural failure due to fatigue especially at pipe nipples, stubs, and instrumentation connections. Fig.1 shows a typical sound spectrum for a compressor we have measured. Various sources at their related frequencies can be depicted and related to a couple of components participating in the noise emission. 


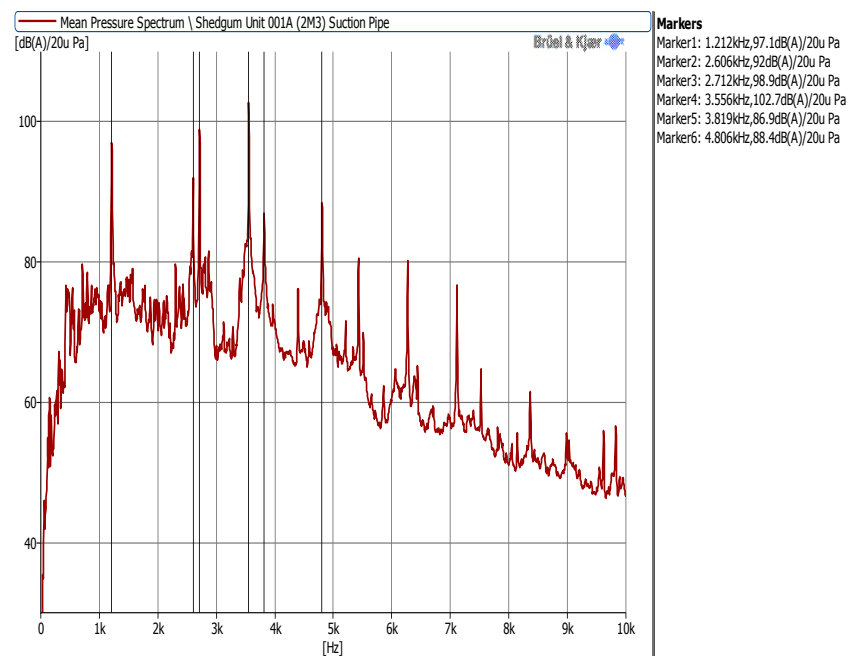

Figure 1: Typical narrow band measured sound pressure level of compressor.

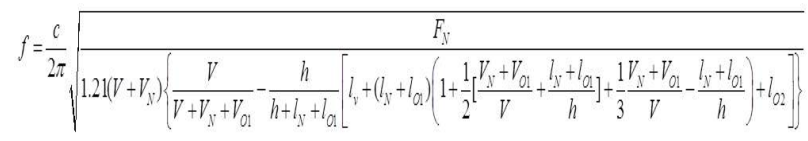

In any centrifugal compressor as the fluid flow exits the impeller, the flow distribution is distorted. Specifically, such distorted flow is characterized by a low angle (relative to a tangent to the impeller circumference) fluid flow exiting most prominently adjacent to the shroud side of the diffuser. In the past, this distorted flow has been shown to cause severe compressor performance problems [5]. Due to the design of the compressor, the inlet and discharge pipes are relatively more susceptible to noise transmission than the compressor casing itself. Noise propagates through the path with least resistance and since the piping at the inlet has thinner walls when compared to the compressor casing, this provides a path of lower resistance for noise propagation. Between the inlet and the discharge, investigations have found that higher vibration and noise levels emanate from the discharge. Noise generated inside the compressor can propagate upstream to the inlet pipe and downstream to the discharge pipe as the flow is typically subsonic inside compressors. Helmholtz resonators consist of a cavity communicating with the main duct through a neck. They have been widely used to effectively attenuate the narrow band low frequency noise. The classic lumped approach is approximates this resonator as an equivalent spring of cavity and mass (neck) system, and yields the expressions for the resonator frequency and the transmission loss [7]. A previous work by the authors [8] has shown also the design of one and two degrees of freedom resonators to evaluate the effect of the size and arrays on the overall noise attenuation performance.

\section{Development of resonator performance}

Analytical analysis can be carried out using form factors shown next to include the effect of geometry. We have demonstrated in [8] that numerical computation using COMSOL, analytical and experimental results were in good agreement. A formula for resonant frequencies was developed in the late nineteenth century to include the effect of the geometry of the resonators[11] and is shown in Eq.(1) where, $f$, resonant frequency, $c$, velocity of sound in the gas, $F_{\mathrm{N}}$, area of the neck, $l_{\mathrm{N}}$, length of the neck, $V_{\mathrm{N}}$, volume of the neck $\left(\mathrm{V}_{\mathrm{N}}=\mathrm{F}_{\mathrm{N}} \times l_{\mathrm{N}}\right), V$, volume of the resonator without the neck, $h$, height of the resonator from the bottom to the neck (see also the next section), $l_{\mathrm{v}}$, form factor, defined in Eq.(2).

$l_{v}=\frac{F_{N}}{V h} \int_{0}^{h} \frac{x V(x)}{F(x)} d x$

here $F(\mathrm{x})$ is the area of a cross-section of the resonator expressed as a function of distance $\mathrm{x}$ from the bottom and

$$
V(x)=\int_{0}^{x} F(\xi) d \xi
$$

$l_{\mathrm{O} 1}, l_{\mathrm{O} 2}$ are two parts of the total end-correction length due to the motion of gas particles outside the resonator. Generally the values $l_{\mathrm{O} 1}=l_{\mathrm{O} 2}=0.24 \mathrm{r}$, where $\mathrm{r}$ is the radius of the neck or opening of the resonator. And $V_{\mathrm{O} 1}$ is the volume of the hypothetical elongation of the neck due to the motion of gas particles outside the resonator $\left(V_{\mathrm{Ol}}=F_{\mathrm{N}} l_{\mathrm{O} 1}\right)$.

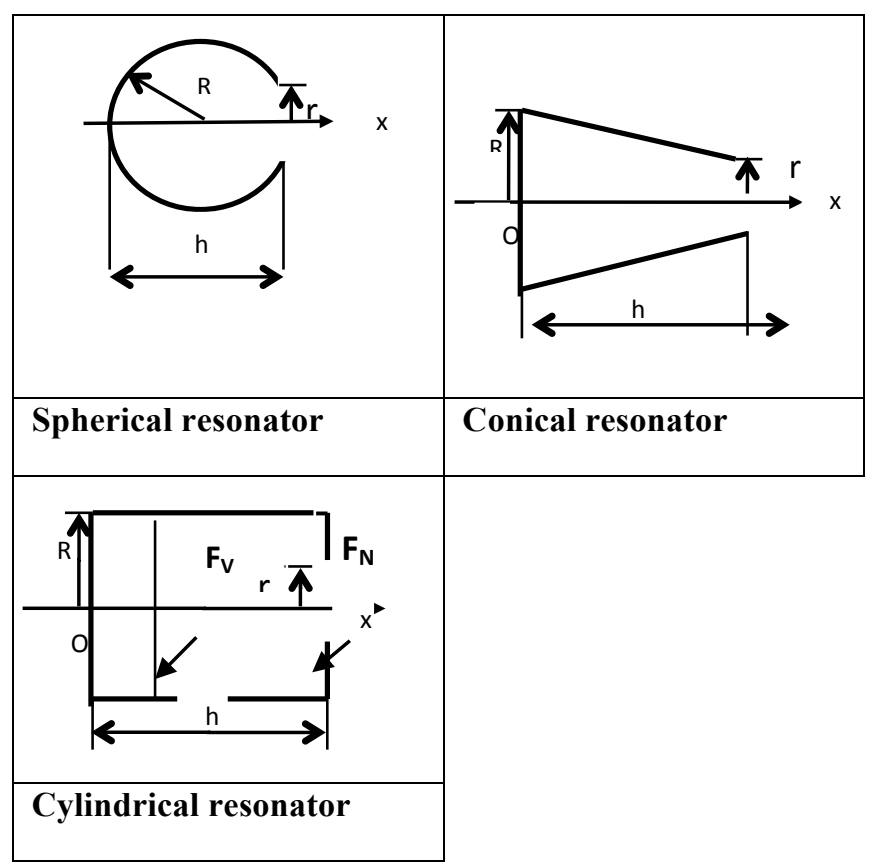

Table 2: Resonator shapes considered in this study. 


\section{Form factor( $\left.l_{v}\right)$ for fundamental forms of volumes [11]}

Form factor $\left(l_{v}\right)$ is determined to calculate the effects of forms of volumes of the resonator. Following are the different form factors for volumes.

\section{Sphere.}

$l_{v}=\frac{r^{2}}{R} \frac{1}{2-A}\left[\frac{1}{3}-\frac{1}{2(A+1)}-\frac{2}{(A+1)^{2}}+\frac{4}{(A+1)^{3}} \ln \frac{2}{1-A}\right]$

where, $A=\sqrt{1-(r / R)^{2}}$

\section{Frustum of a cone.}

$l_{v}=\frac{h}{(R / r)^{2}+(R / r)+1}\left[\frac{1}{3}-\frac{R}{2(R-r)}-\left(\frac{R}{R-r}\right)^{3}\left(\frac{R}{r}-1-\ln \frac{R}{r}\right)\right]$

3. Cylinder.

$$
l_{v}=\frac{F_{N} h}{3 F_{V}}
$$

For an accurate prediction of the resonant frequency in one Degree of Freedom (DOF) cylindrical resonators the following equation (7) can be used [8].

$\omega=c \sqrt{-\frac{3 \cdot L_{n}+L_{c} \alpha}{2 L_{n}{ }^{3}}+\sqrt{\left(\frac{3 \cdot L_{n}+L_{c} \alpha}{2 L_{n}{ }^{3}}\right)^{2}+\frac{3 \alpha}{L_{n}{ }^{3} L_{c}}}}$

where $L_{c}, L_{n}$, a represent the cavity length, the corrected neck length, and $\alpha$ the ratio of cross sections surface of cavity; $a_{c}$ and neck; $a_{n}$, respectively.

The only restriction in the above mentioned formulae is diameter must be less than a wavelength at the resonance frequency. The transmission loss (TL) for one DOF can also $\mathrm{b}$ calculated using the equation [7-10].

$T L=10 \log _{10}\left[1+\left(\frac{a_{n}}{2 \cdot a_{c}} \frac{(1 / \alpha) \tan \left(k \cdot L_{c}\right)+\tan \left(k \cdot L_{n}\right)}{(1 / \alpha) \tan \left(k \cdot L_{n}\right) \tan \left(k \cdot L_{c}\right)-1}\right)^{2}\right]$

Where $\mathrm{k}$ is the wave number. In order to combine the effects of end correction factors the following equations are considered.

$l_{n}=L_{n}-\delta_{1}-\delta_{2}$
An en correction that accounts for the higher ordre wave propagation is [12].

$$
\delta_{2}=0.48 \sqrt{a_{n}}(1-1.25 \sqrt{\alpha})
$$

To account for the higher order wave propagation effects between the circular neck and main pipe (one direction being infinite, while the size of the other direction is close to that of the neck), the end correction is approximated by

$$
\delta_{1}=0.46 \frac{\sqrt{a}_{n}}{2}
$$

\section{Numerical simulations of the various shapes of resonators}

COMSOL was used for the FE Analysis. Each cylindrical duct of diameter $10 \mathrm{~cm}$ and length $100 \mathrm{~cm}$ was fitted with either one or four Helmholtz resonators flush mounted circumferentially at the centre of the duct. The medium of sound propagation was Air. Sound hard boundary wall condition was imposed on all the parts of the duct except inlet boundary and the outlet boundary of the duct where plane wave radiation was considered. The source of sound is a power point source placed at the inlet emitting 5 Watts of (RMS) acoustic power which explains the high level of SPL inside the duct. Same boundary conditions were used for all the four different configurations of the ducts. Tetrahedral elements mesh generating function of COMSOL was used for each duct reaching approximately 1042690 domain elements, 152434 boundary elements, and 10240 edge elements. The size of the elements ranges from $20 \mathrm{~mm}$ to $0.2 \mathrm{~mm}$. Since the Frequency range of interest was under $2 \mathrm{KHz}$, an extremely fine mesh was considered with approximately 7 elements per wavelength which provided a reasonably good estimate of the sound propagation inside the duct.

\subsection{Single resonators}

A single one DOF cylindrical resonator was simulated numerically. Figure 2 shows an empty pipe without resonator and hence no noise reduction, while if a resonator is added a clear noise reduction is observed. A closer view shows perfectly the resonance inside the resonator inducing noise attenuation along the pipeline immediately after the resonator. The front waves are distorted close to the resonator. 


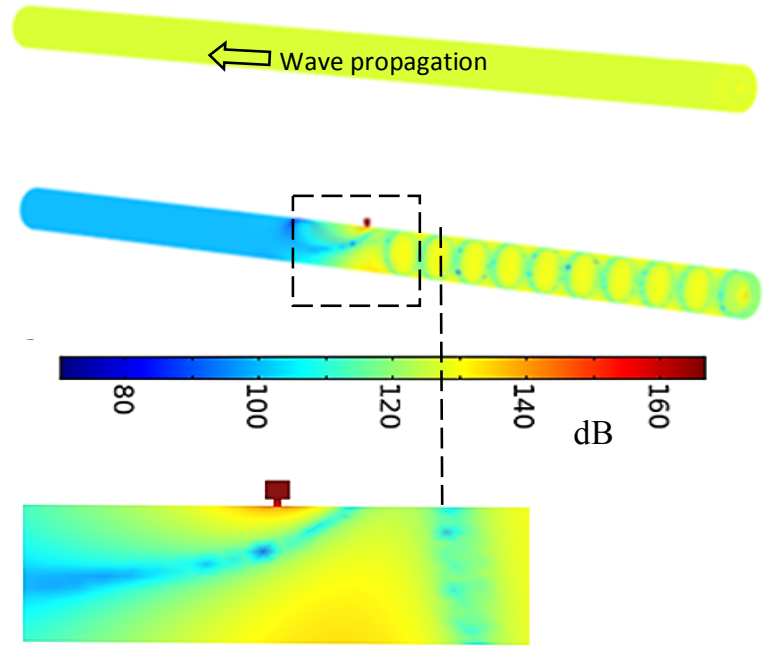

Figure 2: The sound pressure level at $3556 \mathrm{kHz}$ (a) For a pipe without any resonator (b) For a pipe with one 1 DOF cylindrical resonator.

\subsection{Array of resonators}

Rather than considering one resonator, the pipeline could be loaded with several identical resonators around the pipeline cross section. The resonator distribution is indicated in Fig. 3 . The results show an improved noise attenuation compared to the one achieved in Fig. 2 with single attenuation.

In another attempt to investigate the shape effect on the noise reduction, three different shapes of the resonators have been considered. This includes cylindrical, conical and spherical shapes.

The volume of the three cavities was chosen to be equivalent. Numerical simulations have been performed for three various shapes of the resonator. The results shown in Figs 4 to 9 were obtained with three different blade passing frequencies acting at the pipe inlet. The simulations show clear noise reduction for each shape depending on the BPF considered.

Fig.4 shows four resonators mounted in the middle way of the pipe with clear reduction of sound ( $>40 \mathrm{dBA})$ using spherical resonators compared to other shapes under similar conditions with expected results. Fig 6 and Fig 8 depict the resonance phenomenon in cylindrical and conical resonators respectively. In order to have a clear view of the sound pressure level distribution in the pipes Figs 5, 7 and 9 can be referred to for spherical, cylindrical and conical geometries respectively. A simulation was carried out to compare the effect of resonators array on the transmission loss achieved.

Figs. 2 and 6 where two different pipes with a single and an array of four one DOF cylindrical resonators were simulated and compared. It was found that increasing the number has a very limited effect range, increasing the transmission loss by around $5 \mathrm{~dB}$.

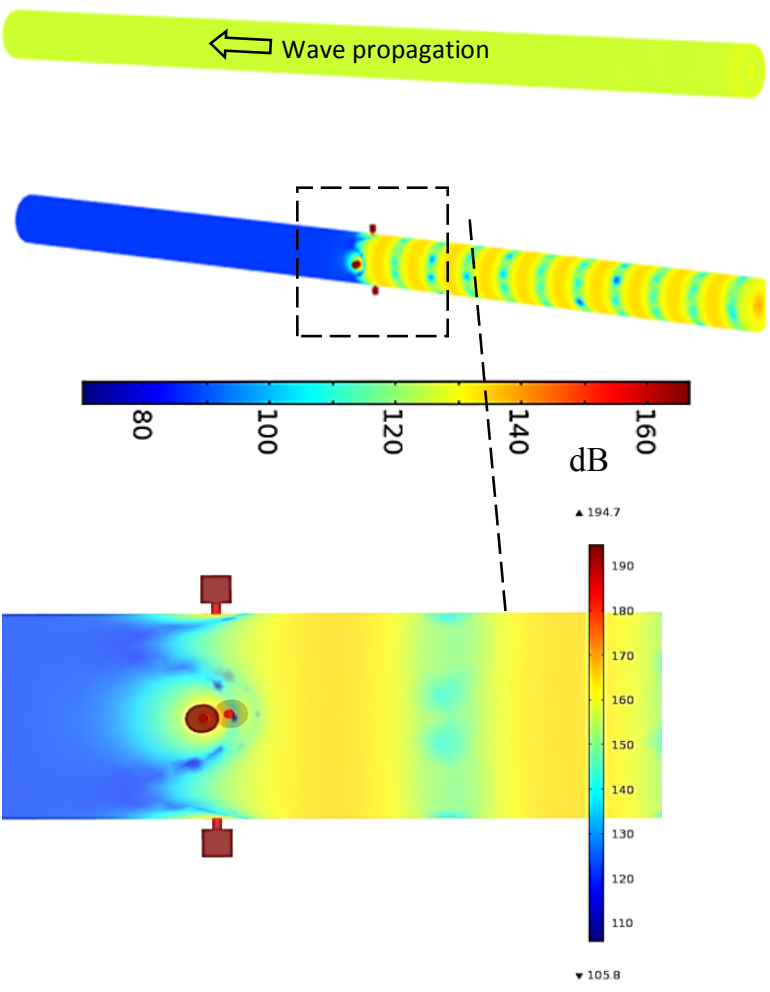

Figure 3: Sound Pressure levels distribution at $3556 \mathrm{~Hz}$ on the surface of the pipe with and without 1 DOF designed array resonators with a closer view of the tuned resonators.

When comparing the results for the two configurations of arrays, i.e. one and four sets of resonators, the frequency for which they are designed doesn't match accurately showing a little difference of around 30-50 Hz. This happens because when array of resonators are put around their resonating frequencies some of them resonate for a particular value while others could not achieve full resonance for that value and this happens due to possibly incomplete air flow filling in the resonators at the same time due to neck size and/or numerical slight precision in positioning different orientations of the resonators on the duct. This phenomenon can be perceived from Fig.9, where different SPL are encountered for a particular designed frequency in the conical resonators, and also in Fig.10 that exhibits sound pressure levels of several configurations treated numerically. Four resonators show better attenuation of sound level compared to single resonator as expected. The numerical results exhibit a frequency shift due possibly to the array of resonators total volume and size affecting the natural frequency in Eq. (1) and Eq. (6).

Another notable observed feature is for one resonator the reduction of noise takes a while which is clear from Fig. 2 , while in the case of four resonators the reduction is almost instant as in Fig. 7. 

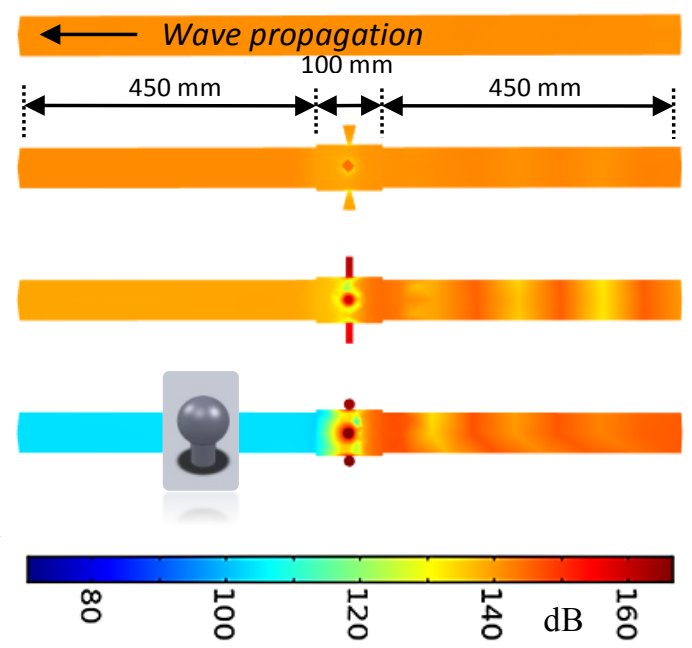

Figure 4: The sound pressure level at $1.284 \mathrm{kHz}$ (a) Pipe without any resonators, (b) Pipe with conical resonators,(c) Pipe with cylindrical resonators, (d) Pipe with Spherical resonators(Spherical resonant frequency).

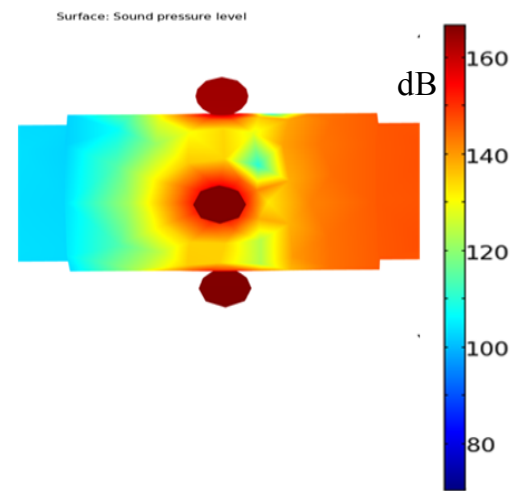

Figure 5: A closer view of the sound pressure level distribution at $1.284 \mathrm{kHz}$ (Spherical resonant frequency). (a)

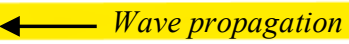

(b)

(c)

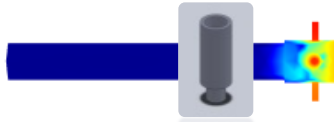

(d)

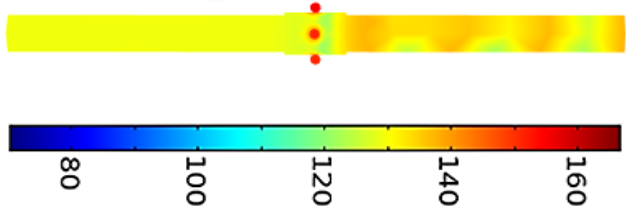

$\mathrm{dB}$

Figure 6: The sound pressure level at $1.15 \mathrm{kHz}$ (a) Pipe without any resonators, (b) Pipe with conical resonators(c) Pipe with cylindrical resonators (Cylindrical resonant frequency), (d) Pipe with Spherical resonators.

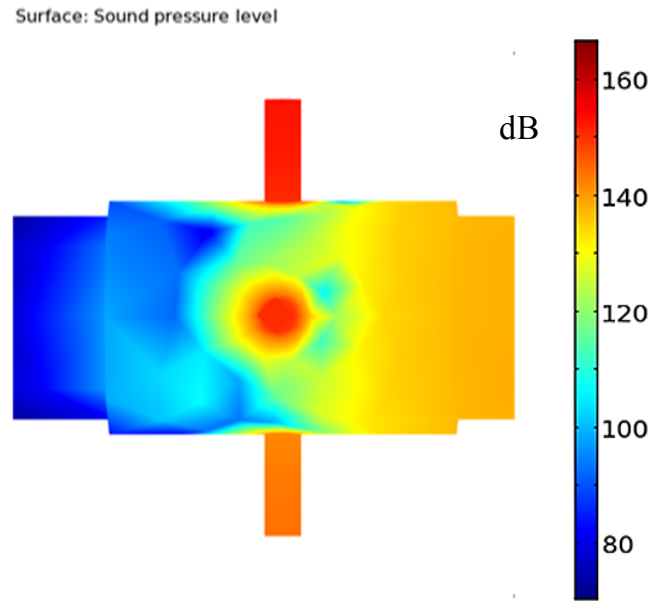

Figure 7: A closer view of the sound pressure level distribution at $1.15 \mathrm{kHz}$ (Cylindrical resonant frequency). 
(a)

(b)

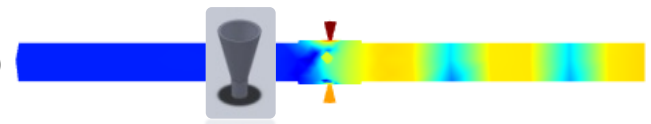

(c)

(d)

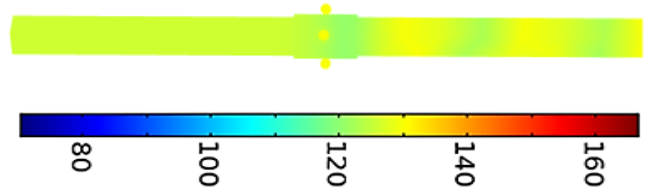

$\mathrm{dB}$

Figure 8: The sound pressure level distribution at $0.84 \mathrm{kHz}$ (a) Pipe without any resonators (b) Pipe with conical resonators (Conical resonant frequency) (c) Pipe with cylindrical resonators (d) Pipe with Spherical resonators.

Fig. 11 represents a numerical comparison of the sound pressure levels of the three different geometries simulated. On careful consideration the resonant frequencies found for cylindrical and conical resonators from the experiments match closely with the frequencies found in the simulations.

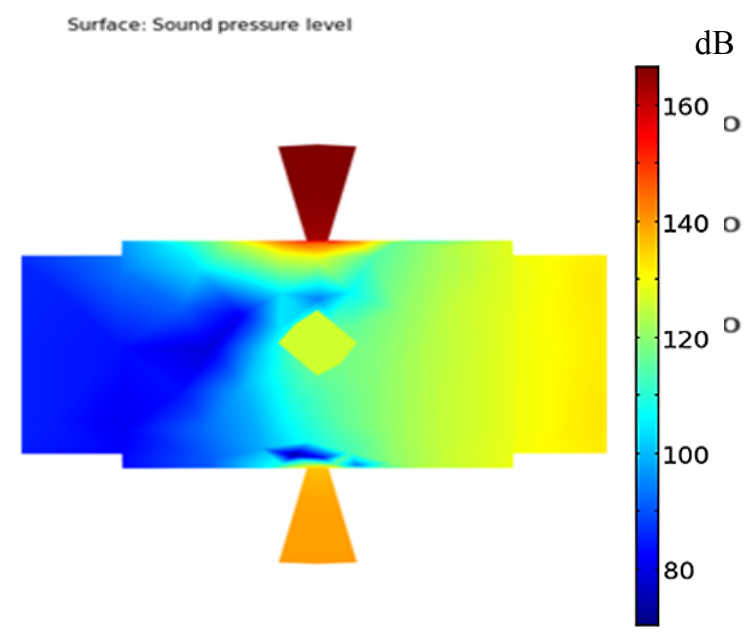

Figure 9: A closer view of the sound pressure level distribution at $0.84 \mathrm{kHz}$ (Conical resonant frequency).

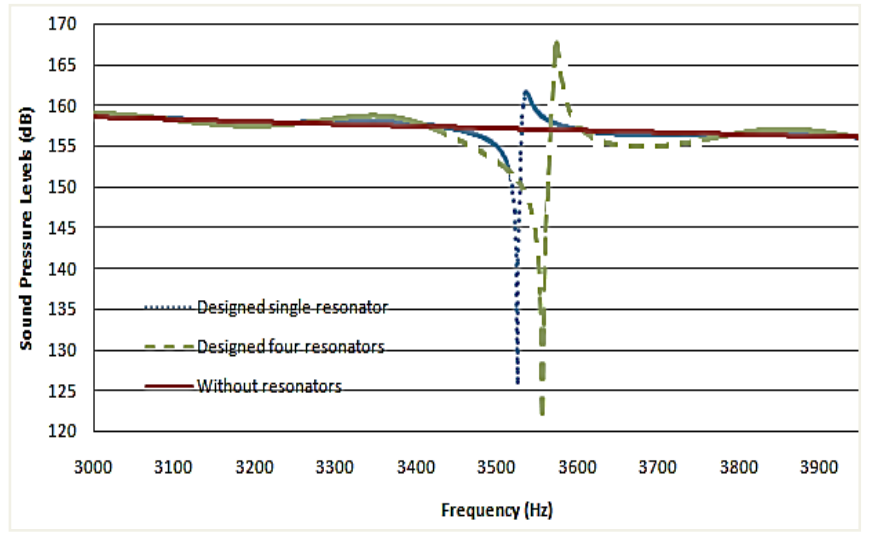

Figure10: Comparison of Transmission Loss with respect to frequency for one and four sets of one DOF cylindrical resonator.

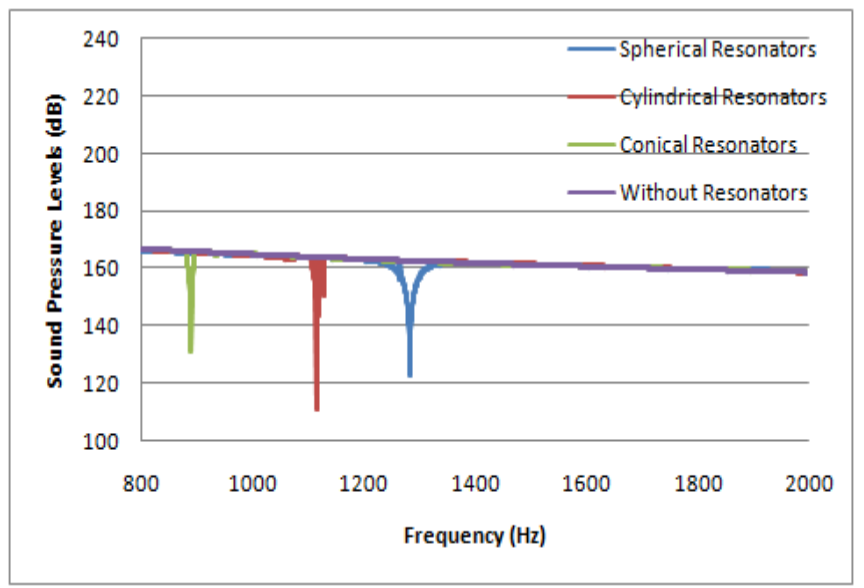

Figure 11: A comparison of the sound pressure levels from the simulations for a pipe fitted with three different arrays of resonators with a pipe without any resonator.

Fig. 11 shows the resonator response over their effective ranges. An anti-resonance behavior was displayed at around $1200 \mathrm{~Hz}$ in cylindrical resonator arrangement and at around 930 in conical resonator arrangement which caused the noise level to amplify by around $3 \mathrm{~dB}$. This phenomenon is not uncommon in such resonator arrangements.

\section{Experimental results and validation}

A one meter straight PVC pipe was cut into two equal parts of $450 \mathrm{~mm}$. Using rapid prototyping process three different geometries of the resonators were manufactured and were fitted on another manufactured polymeric pipe of $100 \mathrm{~mm}$ containing holes as shown in Figs. 13 a) to c). A preliminary test was made using the one meter PVC pipe with no resonators to check the effect of natural damping due to the air itself. The pipe was attached to the insulation and 
mounted on a stand while the generated noise level was varied between 800 to $2000 \mathrm{~Hz}$ on one side of the pipe and similar level was collected on the other end, implying that there was little to no damping within the pipe. Finally the duct tape was added as a precaution to hold it in place. Fig. 12 shows a picture of the experimental set up used for the test. The aim was to find the range over which the resonators are effective along with the resonant frequency of the resonator and gives maximum noise attenuation. Initially the starting frequency was set at $800 \mathrm{~Hz}$.

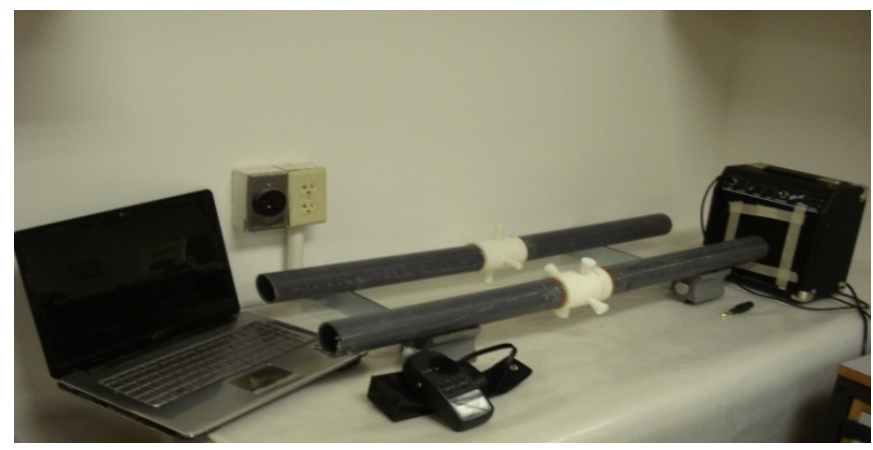

Figure 12: A picture of the experimental setup established to measure the noise attenuation offered by the modeled resonators.
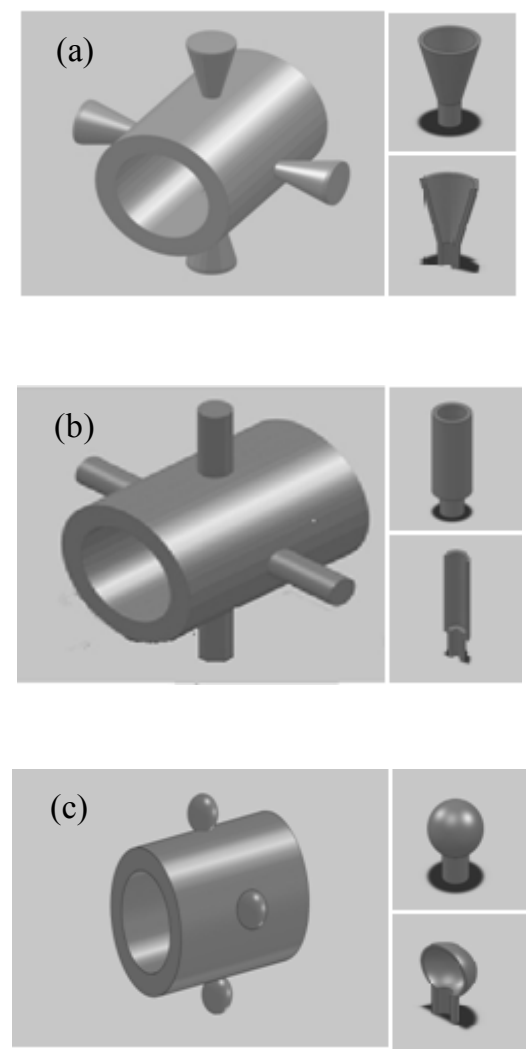

Figure 13: Various resonators Arrangement (a) Conical, (b) Cylindrical, (c) Spherical.
The noise source was a speaker generating a sine wave with maximum SPL of $121 \mathrm{~dB}$ was verified and then the pipe was attached to the noise source. A check was made along the pipe using the sound meter to identify any acoustic leakage. To verify any acoustic leakage, a noise measurement at the outlet was taken. A noise decrease of 2 $\mathrm{dB}$ was observed. Then the process was continued varying the frequency systematically, first increasing at regular intervals and then decreasing, whilst recording the sound level until the noise levels of the source were reached, and consequently no attenuation was found. This has established a range of values around which the resonator provided some level of attenuation. Through varying the frequency it was found that the resonant frequency of the conical arrangement was nearly $840 \mathrm{~Hz}$ at which a reduction of around $8 \mathrm{~dB}$ was observed. The noise level was found to be $106 \mathrm{~dB}$. There was another check made using the noise level meter against acoustic leakages along the pipe and verify the source noise levels, and it was found that such leakages were completely negligible. Next was the testing of the cylindrical pipe arrangement. A similar sweep was performed using the sound meter to check for leakages and it was found that there were minor leakages around the connection region that might tamper with the experimental results. A sleeve made of cotton cloth was made to blanket the noise levels at these locations. The points of leakage were checked and it was found that the cotton cloth successfully blocked any acoustic leakage. On repeating the test for cylindrical resonators the resonant frequency was found to be approximately $115 \mathrm{~dB}$. The spherical case couldn't be tested since the spherical resonators had pores due to some defects in the rapid prototyping process during their manufacturing.

In the numerical investigation the spherical resonators are also found to have the same behavior as predicted from the analytical results. The noise reduction achieved in the experiments are less than those achieved in the COMSOL simulations which could be due to following reasons: One dimensional propagation is assumed in the simulations which can be attenuated more easily than the actual three dimensional propagation in the experiments, improper acoustics terminations at the open ends, damping offered by the polymeric material and the PVS pipe due their acoustic absorption coefficients. A slight shift in the natural frequency may be due to the fact that the volume was not anymore equivalent due to rapid prototyping inaccuracy of the cavities. 


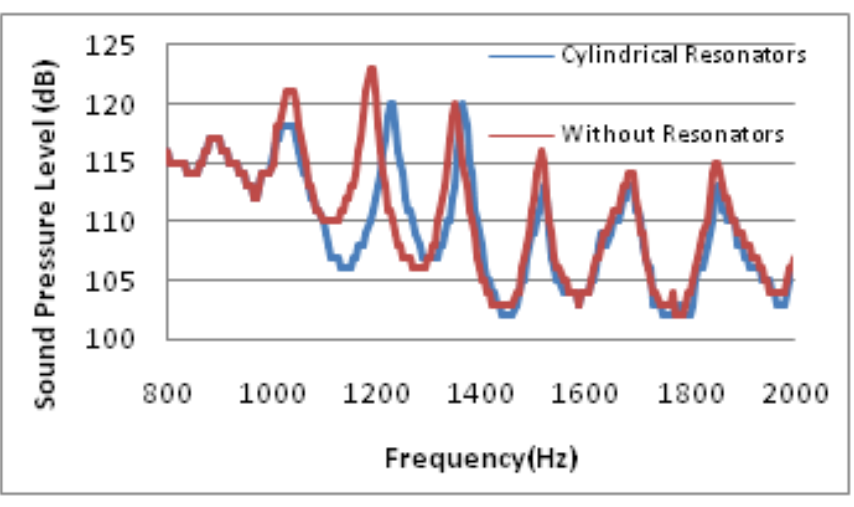

Figure 14: A comparison of the sound pressure levels from the experiments for a pipe fitted with an array of cylindrical resonators with a pipe without any resonator.

\section{Conclusion}

A numerical simulation validated by analytical method and experimental tests to estimate the level of noise attenuated using Helmholtz resonators as an add-on solution to pipeline has been presented in this paper. The method was used to analyze the effects of the various shapes e.g. cylindrical, conical, and spherical on the noise reduction in pipelines. The effect of number of resonators has also been studied and presented.

Comparison tests between various shapes of the resonator have shown in both numerical and experimental methods that cylindrical resonators give better noise attenuation than the conical and the spherical resonators. The three different geometries have distinct resonant frequencies and transmission loss even though the volume for all the cases is equal (Figs 11 and 14). Some of the noted effects of number of resonators are when using one resonator the reduction of noise takes a while, but in the case of four resonators the reduction is almost instant. Also the increase in transmission loss achieved by increasing the number of resonators from one to four has a very limited effect range, increasing the transmission loss by around $5 \mathrm{~dB}$.

Further investigations will be considered based on these findings to refine the parametric design and investigate the effect of size for example. Different sizes of sound absorbing materials can also be experimented and there by checked for the noise attenuation for comparison purposes. It is recommended that the analytical equations for the different geometries have a considerable room for improvement in accuracy and should be given due consideration even though their manufacturability is a tough task.

\section{Acknowledgments}

The authors would like to acknowledge the support provided by the research collaboration between King Fahd University of Petroleum\& Minerals (KFUPM) and DresserRand Company (USA).

\section{References}

[1] Zheji Liu and Mark J. Kuzdzal, Noise Control Of An 11,000 Horsepower Single Stage Pipeline Centrifugal Compressor, Proceedings of ASME Turbo Expo 2007 Power for Land, Sea, and Air, May 11, 2007

[2] Zheji Liu and Mark J. Kuzdzal, Noise Reduction Of A Multistage Export / Reinjection Centrifugal Compressor Through The Use Of Duct Resonator Arrays, Dresser-Rand Company Olean USA, 2002

[3] Till Raitor, Wolfgang Neise, Sound generation in centrifugal compressors, Journal of Sound and Vibration, Volume 314 , Issues 3-5, 22 July 2008, Pages 738-756

[4] It All Boils Down To Two Things www.wgs4.com.

[5] W. Neise, G.H. Koopmann, Reduction of centrifugal fan noise by use of resonators, Journal of Sound and Vibration, Volume 73, Issue 2, 22 November 1980, Pages 297-308

[6] Kim Jeung, Imam Imdad. "Compressor Noise Attenuation Using Branch Type Resonator". Patent 4,927,342. May 22, 1990.

[7] Mekid S. and Farooqui, M. Design of Helmholtz resonators in one and two degrees of freedom for noise attenuation in pipelines, Acoustics Australia, Vol. 40 No 3, 2012.

[8] Munjal MI, Acoustics of ducts and mufflers with application to exhaust and ventilation system design, New York Wiley, 1987.

[9] Selamet A, Radavich PM, Dickey NS, Novak JM.Circular concentric Helmholtz resonators. JAcoSoc Am 1997, 101:4151.

[10] Li, D. (2003). Vibroacousticbehaviour and noise control studies of advanced composite structures. $\mathrm{PhD}$ thesis, University of Pittsburgh.

[11] M.Alster. Improved calculation of resonant frequencies of Helmholtz resonators Journal of sound and vibration 1972, 24(1) 63-85.

[12] Selamet A, Lee I-J. Helmholtz resonator with extended neck. J AcoustSoc Am2003; 113:1975-85. 